\title{
Editorial
}

\section{Technical Assistance to Tuberculosis Control Programmes as a Platform for Infectious Disease Research}

\author{
Frank van Leth ${ }^{*}, 1, \S$ and Peter Gondrie ${ }^{2}$
}

\author{
${ }^{I}$ Department of Global Health, Academic Medical Center, University of Amsterdam, Amsterdam Institute for Global \\ Health and Development, The Netherlands
}

${ }^{2}$ KNCV Tuberculosis Foundation, The Hague, The Netherlands

The topic of this Supplement of The Open Infectious Diseases Journal might come as a surprise to some of its readers. What does Technical Assistance (TA) to National Tuberculosis Programmes (NTPs) has to do with the scope of a journal that "publishes articles in all areas of clinical and experimental infectious diseases research"?

National disease-control programmes, like that for tuberculosis, in countries with a high burden of disease are often organized in a vertical manner. Clinical management of individual patients in such a set-up is strongly driven by topdown implementation of a set of guidelines embedded in a hierarchical supervision structure. As such, the strength and the organization of a national disease-control programme has a direct bearing on the content and the quality of clinical care provided.

Many NTPs are supported by Technical Agencies. Rimer et al. describe an effective TA system as an interplay between "pull and push", in which pull refers to an assistance request from national programmes to TA organizations based on identified needs, and push refers to pro-active integration of new knowledge or technology into the programmes by TA organizations [1]. Within this framework, scientific evidence on clinical management as well as service delivery is dependent on a good interplay between NTPs and TA organizations. This makes that everyday clinical practise, programmatic disease control activities, and TA-activities are intertwined rather than opposed to each other.

KNCV Tuberculosis Foundation (KNCV) was created in 1903 for control of TB in The Netherlands. Since the eighties of the $20^{\text {th }}$ century it provides also TA to NTPs world-wide. Currently the organization supports about 30 programmes mainly in sub-Saharan Africa and Asia, but also in Eastern Europe and Latin-America. This assistance is preferably through building long-term working relationships

*Address correspondence to this author at the Department of Global Health, Academic Medical Center, University of Amsterdam, Amsterdam Institute for Global Health and Development, Pietersbergweg 17, 1105BM Amsterdam, The Netherlands; Tel: +31 20566 1593;

E-mail: f.vanleth@aighd.org

${ }^{\S}$ Guest Editor. rather than one-off contractual assignments, to ensure adequate knowledge of the local context and differential needs of the NTP over time. To complement the work of the general TA consultants, KNCV instituted a research unit to accommodate the need for evidence-based solutions to programmatic obstacles. Epidemiologists work in close collaboration with the general TA consultants as well as within the international TB-research community at large. This results in strong research support embedded in the longterm cooperation between NTPs and KNCV. West et al. showed that such a set-up can meet the two critical functions of an ideal TA system: incorporation of in-depth knowledge of the needs of the programme, and continuous evaluation of emerging knowledge and determination of which is relevant to the programmes to which TA is provided [2].

The articles in the Supplement highlight some of the TA activities of KNCV. It brings the TA activities and their results from the "grey" unpublished literature, where the reports most often end up, into the public domain. It also gives insight into the often "concealed" relationship between clinical practise and public health, which is influenced by the effectiveness of NTPs and their TA organizations.

The first two papers describe classical TA activities. Van Gorkum and colleagues present data from Namibia, in which they show how a long-term collaboration between the NTP and KNCV improved TB-control activities in the country. This as a result of mobilizing external funding, improving staffing within the health sector, training of health staff at different levels, and improving patient care. The authors, however, conclude with the observation that much of the gains made in the last 10 years can be lost if continuous funding cuts leads to unacceptable budget constraints within the NTP. Shimeles and colleagues describe how a rapid response to the identified need for addressing the emergence of multi-drug resistant (MDR) TB in Ethiopia led to guideline development, access to drugs, and improved clinical management. The authors stress the need for adequate political commitment and strong collaborations between partner organizations.

The following five papers describe typical research projects within crucial areas of TB-control: case-finding, TB-HIV co-infection, service delivery, and epidemiology. Tiemersma and colleagues illustrate how the long-term 
collaboration between the NTP of Vietnam and KNCV provided a scientific discourse on TB case finding, leading to a clearly formulated hypothesis and subsequent study to test this hypothesis. The authors show that the increase in TB case notification seen in young adults is unlikely to be the result of immigration. By refuting this hypothesis the research team contributed to the on-going discussion on why Vietnam does not see the expected decline in TB-incidence as expected by the good performance indicators for the NTP. Mitchell and colleagues describe the results of a study using the approach of "mystery client" as a way to assess the impact of training in proper case identification and referral by pharmacists and corner shop keepers. The authors show that a simple motivational intervention can improve referral of individuals with TB symptoms to appropriate TB diagnostic facilities. This finding is of importance especially in countries with a large informal health sector. Tursynbayeva and colleagues report on the collaboration between the TB and HIV programme in Kazakhstan. The authors show that recording of TB-HIV co-infected patients is not optimal in either of these programmes, precluding proper case management. They emphasize the need for better collaboration to improve patient care. In another report from Kazakhstan, Kaliakbarova and colleagues describe the effects of providing psychological and social support to patients with MDR-TB to curb the high rates of treatment default. The authors show that such type of support has a positive effect on correct drug intake, and decreases overall default rates. However, the authors identified the need for the development of standardized tools to monitor the effects of psycho-social support. Finally, van Leth and Klinkenberg give an overview of population-based surveys to obtain epidemiological estimates of the burden of disease. Epidemiological parameters are essential indicators for the impact that TB-control programmes have. The authors discuss the main areas for TA in these surveys and describe the lessons learned. The authors conclude that epidemiological research conducted in the context of longterm partnership between NTP and TA organization promotes uptake of the results and improves programme strength.

This Supplement ends with a paper on the impact of capacity building in TA activities. Mitchell and colleagues discuss the effect of training activities in the context of implementing TB-HIV activities in Kenya. The authors analyzed data from the Kenya Service Provision Assessment including over 1000 service providers from over 400 health facilities. The results show that TB training interventions through TA-activities are significantly associated with improved performance in key areas of TB-control. The authors put a note of caution by stating that this result can only be sustained in a vital health system with respect to human resource capacity and health system management.

We hope that the readers of The Open Infectious Disease Journal will enjoy this Supplement, and that it may serve as an illustration of the strong connections between clinical management and public health. By bringing descriptions of TA-activities, their results, and their impact into the public domain, we want to contribute to the scientific discussion on the content and the effectiveness of these activities.

\section{REFERENCES}

[1] Rimer BK, Glanz K, Rasband G. Searching for evidence about health education and health behavior interventions. Health Educ Behav 2001; 28: 231-48.

[2] West GR, Clapp SP, Averill EMD, Cates W Jr. Defining and assessing evidence for the effectiveness of technical assistance in furthering global health. Glob Public Health 2012; 7 : 915-30.

(C) van Leth and Gondrie; Licensee Bentham Open.

This is an open access article licensed under the terms of the Creative Commons Attribution Non-Commercial License (http: //creativecommons.org/licenses/by$\mathrm{nc} / 3.0 /$ ) which permits unrestricted, non-commercial use, distribution and reproduction in any medium, provided the work is properly cited. 